\title{
Die Sol-Gel-Umwandlung von gereinigtem Aktomyosin und von Aktomyosin während der Extraktion frischer und glycerinbehandelter Muskelfasern in Abhängigkeit von Ionalität, Ionenstärke und ATP*
}

\author{
Von W. Hasselbach, H. P. Hofschneider, E. Kasper und R. Lutz \\ Aus dem Physiologischen Institut der Universität Tübingen (Direktor: H. H. W e b e r) \\ (Z. Naturforschg. 8 b, 204-211 [1953]; eingegangen am 23. Februar 1953)
}

1. Es wird die Löslichkeit von Aktomyosin in einem Intervall der Ionenstärke von etwa $0,1-0,4 \mu$ mit und ohne ATP studiert, um präzise Daten über den Übergang aus dem kontraktionsfähigen Gel - in den dissoziationsfähigen Solzustand zu bekommen. Die Löslichkeit und Fähigkeit zur Superpräcipitation in Lösungen verschiedener Ionalität werden dargestellt als Abhängigkeit dieser Eigenschaften von der Ionenstärke der Systeme.

2. Ohne ATP hängt die Löslichkeit gereinigten Aktomyosins außer von der Ionenstärke nur vom $p_{\mathrm{H}}$ ab.

3. Sie hängt von der Ionenstärke völlig gleichartig $a b$, wenn ein Teil der K-Ionen durch $\mathrm{Mg}++$ oder $\mathrm{Ca}^{++}$ersetzt wird (bis zu einer Ionenstärke der Erdalkalien von 0,09 $\mu$ ). Dasselbe gilt für den Ersatz des Cl-Ions durch Pyrophosphat bis zu einer Ionenstärke von 0,26 u. Ein weitergehender Ersatz wurde nicht geprüft.

4. Die Abhängigkeit der Gel-Sol-Umwandlung von der Ionenstärke wird ebenfalls (zwischen $0^{\circ}$ und $20^{\circ} \mathrm{C}$ ) nicht von der Temperatur und auch nicht vom Alter der Aktomyosinlösung beeinflußt. Die Umwandlung erfolgt immer zwischen 0,2 und $0,3 \mu$.

5. Dagegen verschiebt sich der Umwandlungsbereich sehr erheblich zu niedrigen Werten der Ionenstärke, wenn ATP zugegen ist. In Anwesenheit von 5·10-3-m. ATP sinkt die Ionenstärke der Umwandlung um etwa $0,1 \mu$. Der Umwandlungsbereich reicht dann von etwa $0,1-0,2 \mu$ statt von $0,2-0,3 \mu\left(p_{\mathrm{H}} 7\right)$.

6. Dagegen hört die Fähigkeit der Superpräcipitation in Gegenwart von 5.10-3-m. ATP bereits bei etwa $0,15 \mu$ auf und beträgt auch bei $0,08 \mu$ erst ein Drittel des Wertes, der bei dieser Ionenstärke mit $1 \cdot 10^{-3}-m$. ATP gefunden wird. Erhöhung der ATP-Konzentration setzt die Superpräcipitation bereits bei so niedrigen Ionenstärken herab, daß eine Erhöhung der Löslichkeit noch nicht spürbar ist. Außerdem wird die Superpräcipitation - im Gegensatz zur Löslichkeit - bereits durch 0,003-m. $\mathrm{Mg}++$ beeinflußt: Sie wird in 5·10-3-m. ATP-Lösungen durch $\mathrm{Mg}^{++}$noch weiter herabgedrückt.

7. Also beruht die Änderung der Superpräcipitationsfähigkeit und der fermentativen Eigenschaften bei der Gel-Sol-Umwandlung nicht darauf, daß das Gel in Lösung geht. Es gibt Ionalitäten, bei denen das Gel noch nicht gelöst ist, aber schon unfähig ist zur Superpräcipitation, und bei denen die ATPase-Aktivität ebenfalls stark gemindert ist.

8. Der Anteil des Aktomyosins, der in ATP-Anwesenheit gelöst wird, ist immer dissoziiert selbst wenn die Ionenstärke der Lösung unterhalb von $0,15 \mu$ liegt.

9. Die Extrahierbarkeit des Aktomyosins aus den hochkonzentrierten Strukturen der frischen Muskelfaser mit ihrem ATP-Gehalt $\sim 1 \cdot 10^{-3}-m$. (Brei zu ATP-freier Extraktionslösung $=1: 4$ ) ist bei $0,2 \mu$ merklich und bei $0,4 \mu$ erschöpfend, falls der Muskel mit dem Blendor zerkleinert ist.

10. Wenn die Extraktionslösung 6-10-3-m. ATP enthält, sind bereits bei $0,12 \mu 40 \%$ des Aktomyosins extrahierbar.

11. Aus dem geblendorten Brei wird das Myosin als Aktomyosin extrahiert $\left(p_{\mathrm{H}} 7\right)$. Nach Latapiezerkleinerung wird die extrahierte Menge kleiner, und es handelt sich um fast reines L-Myosin.

12. In 27-proz. Glycerinlösung hergestellte Modellfasern geben bei niedriger und mittlerer Ionenstärke viel weniger Aktomyosin ab. Erschöpfende Extraktion ist erst durch Ionenstärken $\geq 1,2 \mu$ möglich. Selbst bei dieser Ionenstärke können dann nur $80 \%$ des Aktomyosins extrahiert werden, wenn die Fasern zusätzlich noch 5 Tage in 80-proz. Glycerin aufgehoben sind.

13. Die Abhängigkeit der Löslichkeit vom Alter der Präparate und der Vergleich mit der Löslichkeit des ATP-freien gereinigten Aktomyosins zeigt, daß diese Löslichkeitsminderung

* Mit Unterstützung des Unitarian Service Committee und des Oberlaender Trust, Philadelphia. 
nicht allein auf ATP-Mangel beruht, sondern auch auf einer Erhöhung der Kohäsionskräfte während der Vorgeschichte.

14. Diese Synaerese-Wirkungen können durch 0,016- $m$. Pyrophosphat ganz oder weitgehend beseitigt werden: Das Aktomyosin der Glycerinfaser ist dann wieder bei 0,6 $\mu$ voll extrahierbar.

15. In Gegenwart von $8 \cdot 10^{-3}-m$. ATP sinkt die Ionenstärke, bei der das Aktomyosin voll extrahiert werden kann, wieder auf etwa den gleichen Wert wie bei der Extraktion frischen Muskels unter gleichen Bedingungen. Die Aktomyosinlöslichkeit scheint unter diesen Bedingungen der Löslichkeit des gereinigten Myosins ungefähr gleich zu sein.

E s ist seit langem bekannt, daß sich das Aktomyosin-Gel durch Einsalzen in ein Sol verwandelt, wenn die Ionenstärke bei $p_{\mathrm{H}} \sim 7$ einen Wert von $0,3 \mu$ überschreitet ${ }^{1,1 a}$.

Es ist ferner bekannt, daß dieser Úbergang aus dem Gel- in den Solzustand die Reaktionsweise und den strukturellen Zustand des Proteinkomplexes Aktomyosin tiefgreifend verändert ${ }^{2}$.

1. Aus der Aktomyosin-ATPase wird dabei die viel weniger wirksame und in ganz anderer Art aktivierbare und hemmbare L-Myosin-ATPase.

2. Das Aktomyosin wird durch ATP zur Schrumpfung, Superpräcipitation und, falls das Gel geordnet ist, zur Kontraktion gebracht. Das Sol erleidet dagegen unter ATP Veränderungen ganz anderer Art, die im allgemeinen als Dissoziation angesehen werden.

Der Übergang der Aktomyosin-ATPase in die LMyosin-ATPase und der Übergang vom superpräcipitierenden zum dissoziierenden Effekt des ATP hängt außer von Ionenstärke und $p_{\mathrm{H}}$ stark von anderen Faktoren ab: ATP, $\mathrm{Mg}^{++}, \mathrm{Ca}^{++}$, Temperatur, Aktinanteil des Aktomyosins und gewissen Struktureigentümlichkeiten des Aktomyosins selbst (s. unten).

Wie stark der Einfluß dieser Faktoren ist, erhellt aus folgenden Zahlenangaben über den Einfluß der ATP und Eiweiß-Struktur. Im lockeren Gel aus gereinigtem Aktomyosin beginnt die Verwandlung der Aktomyosin-ATPase in die L-Myosin-ATPase bei Anwesenheit physiologischer ATP-Konzentrationen bereits bei einer Ionenstärke von $0,1 \mu$ und ist etwa bei $0,2 \mu$ beendigt.

Dagegen zeigen die von Perry ${ }^{3}$ isolierten eiweißreichen Aktomyosin-Fibrillen des Muskels noch bei $0,20 \mu$ die Eigenschaften einer völlig reinen Aktomyosin-ATPase.

Sogenannte Fasermodelle (Glycerin-Wasser-extrahierte Muskelfasern) kontrahieren sich unter ATP bis

$1 \mathrm{H}$. Portzehl, G. S chram u. H. H. W e b e r, Z. Naturforschg. 5 b, 61 [1950].

1a A. Szent Gy örgyi, Stud. Inst. med. Chem. Univ. Szeged 3, 86 [1943].

2 W. H a s s e l b a c h, Z. Naturforschg. 7 b, 164 [1952]. zu Ionenstärken von $\sim 0,4-0,5 \mu^{4}$. Wird ihre Struktur dagegen vor der Zugabe des ATP durch Behandlung mit Pyrophosphat oder Bicarbonat aufgelockert, so kontrahieren sie sich nur, solange die Ionenstärke unter $0,1-0,2 \mu$ bleibt, und die Kontraktion der Aktomyosin-Fäden findet von vornherein nur bei solchen niedrigen Ionenstärken statt ${ }^{5}$.

Aktomyosin zeigt also die fermentativen und kontraktiven Eigenschaften des Geles mit Sicherheit nur bei Ionenstärken $<0,1 \mu$, und es zeigt die Eigenschaften des Soles mit Sicherheit nur bei Ionenstärken $\geqq 0,5 \mu$. In dem Zwischenbereich der Ionenstärke hängt es von den speziellen Bedingungen $a b$, wie weit die Sol- oder Gel-Eigenschaften des Aktomyosins überwiegen. Gerade aber in diesem Zwischenbereich liegt die physiologische Ionenstärke, die auf Grund der Asche zu etwa 0,16 $\mu$ und unter Einrechnung der organischen Ionen - vor allem des ATPat - zu $\sim 0,24 \mu^{5 a}$ angesetzt werden muß.

Es erscheint infolgedessen wichtig, zu wissen auch im Hinblick auf den Zustand des Aktomyosins im lebenden Muskel -, 1. unter welchen Bedingungen das Aktomyosin zwischen 0,1 und $0,6 \mu$ gelöst oder nicht gelöst ist, und 2 . wie weit der Übergang in Lösung identisch ist mit dem Verschwinden der Superpräcipitation (auf ATP-Zusatz) und mit der Änderung der fermentativen Eigenschaften.

Es wird infolgedessen die Abhängigkeit der Löslichkeit von der Ionenstärke unter systematischer Variation der anderen Faktoren untersucht und mit der Fähigkeit zur Superpräcipitation unter ATP verglichen.

Um den Einfluß der Aktomyosin-Struktur selbst kennenzulernen, wird außerdem bestimmt, wieviel Eiweiß unter den gleichen oder ähnlichen Bedingungen aus frischen und aus mit Wasser-Glycerin vorbehandelten Muskelfasern in Lösung geht.

3 S. V. P e r ry, Biochem. J. 48, 257 [1951].

4 A. W e b e r, unveröffentl. Versuche.

5 A. E. Szent Györgyi, Enzymologica [Amsterdam] 14, 246 [1950].

5a M. D u d u is s o n, Arch. int. Physiol. 4, 439 [1942]. 
II.

Werden Aktomyosin-Lösungen aus 24-stdg. Muskelextrakt von Kaninchen mehrfach fraktioniert umgefällt, so erhält man reine Aktomyosin-Lösungen frei von L-Myosin. Konzentrierte Stammlösungen $(\sim 1 \%)$ von Aktomyosin werden auf $0,1-0,2 \%$ verdünnt und hierbei auf bestimmte Ionenstärken, $p_{\mathrm{H}^{-}}$

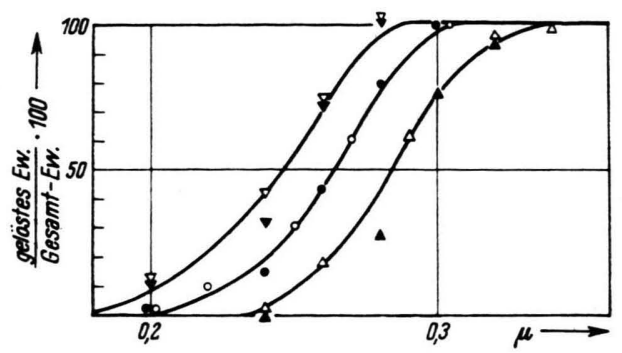

Abb. 1. Die Abhängigkeit der Aktomyosin-Löslichkeit von der Ionenstärke; bei $p_{\mathrm{H}} 6,7$ ^ nur mit $\mathrm{KCl}, \triangle \mathrm{KCl}$ + 0,03-m. Mg; bei $p_{\mathrm{H}} 7,0 \bullet$ nur mit $\mathrm{KCl}, \circ \mathrm{KCl}+0,03-m$. $\mathrm{Ca}$; bei $p_{\mathrm{H}} 7,3$ - nur mit $\mathrm{KCl}, \nabla \mathrm{KCl}+0,03-m$. Mg. Eiweißkonzentration: $0,1 \%, \mathrm{~T}=20^{\circ} \mathrm{C}$.

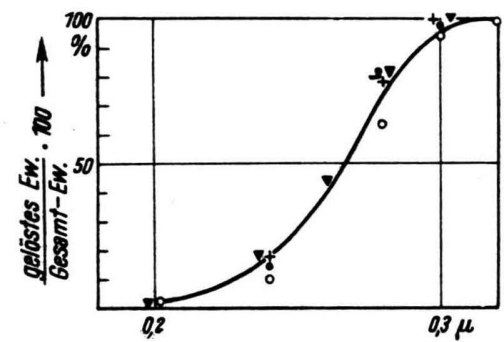

Abb. 2. Die Abhängigkeit der Aktomyosin-Löslichkeit von der Ionenstärke bei $p_{\mathrm{H}} 7,0$ in KCl-Lösung; a) bei $20^{\circ} \mathrm{C}$, Aktomyosin: - 1 Tag, +3 Tage, -9 Tage nach der Präparation, b) bei $0^{\circ} \mathrm{C}$, ${ }^{\circ} 1$ Tag nach der Präparation. Eiweißkonzentration: $0,1 \%$.

Werte und Erdalkali-Konzentrationen gebracht. Es wird geprüft, wieviel an Aktomyosin unter den jeweiligen Versuchsbedingungen in Lösung bleibt.

Es zeigt sich, daß der lösliche Anteil von der Ionenstärke und vom $p_{\mathrm{H}}$ abhängt (Abb. 1). Der lösliche Anteil ist dagegen unabhängig davon, wieweit diese Ionenstärke auf Alkali- oder Erdalkalisalzen beruht - wenigstens bis hinauf zu einer Erdalkali-Konzentration von $0,03-$ molar. Mit wachsender Ionenstärke und mit wachsendem $p_{\mathrm{H}}$ wird die Löslichkeit größer - , und zwar so, daß die Aktomyosin-Fällung bei $p_{\mathrm{H}} 6,7$ in einer Lösung von $0,34 \mu$ beginnt und von $0,24 \mu$ beendet ist. Bei $p_{\mathrm{H}} 7$ sind die entsprechenden Werte der Ionenstärke 0,3 und $0,2 \mu$ und bei $p_{\mathrm{H}} 7,3$
$0,28 \mu$ und $0,18 \mu$. Die Löslichkeit ist außerdem gleich bei $0^{\circ} \mathrm{C}$ und $20^{\circ} \mathrm{C}$. Sie ist schließlich auch davon unabhängig, ob die Aktomyosin-Lösung 1, 3 oder 9 Tage alt ist (Abb. 2).

III.

Wieweit die außerordentliche Verschiedenheit der Wechselwirkung zwischen ATP und Aktomyosin (der ATPase-Wirkung, Superpräcipitation, Dissoziation) bei niedriger und bei hoher Ionenstärke auf dem Übergang aus dem Gel- in den Sol-Zustand beruht, kann nur durch Löslichkeitsstudien in Anwesenheit von ATP geprüft werden. Gewisse Wechselwirkungen zwischen Aktomyosin und Pyrophosphat sind den Wechselwirkungen zwischen ATP und Aktomyosin gleich.

Auch Pyrophosphat (PP) ist für das AktomyosinGel bei Temperaturen bis zu $20^{\circ} \mathrm{C}$ ein Weichmacher, auch PP bewirkt in Aktomyosin-Lösungen hoher Ionenstärke jene Viskositätsphänomene, die als Dissoziation gedeutet werden - aber nur bei Temperaturen nahe $0^{\circ} \mathrm{C}$. Auch PP beschleunigt und verbessert in Extraktionssystemen hoher Ionenstärke die Extraktion des Aktomyosins bei Temperaturen $\sim 0^{\circ} \mathrm{C}$. Es erscheint also zweckmäßig, den Einfluß der Polyorthophosphate auf die Löslichkeit des Aktomyosins nicht nur in ATP-haltigen, sondern auch in PP-haltigen Ansätzen zu studieren.

Die Versuchsbedingungen sind ohne weiteres scharf definiert in PP-Ansätzen, weil PP nicht gespalten wird. In ATP-Ansätzen dagegen muß die Versuchsdauer möglichst kurz und die Proportion vom Aktomyosin und Volumen der ATP-haltigen Lösung möglichst klein gehalten werden, wenn sich die ATP-Konzentration während des Versuches nicht durch Spaltung allzu sehr ändern soll. Auf Grund unserer Kenntnisse über die Größe der Spaltungsrate des ATP in Abhängigkeit von der Temperatur, der Ionenstärke, der $\mathbf{M g}++$-Konzentration und der ATP-Konzentration ${ }^{2}$ ist es möglich, die Spaltungsrate für jeden einzelnen Ansatz der verschiedenen Lösungsserien voraus zu berechnen und die Versuchsdauer und den Eiweißgehalt der Ansätze so einzustellen, daß sich die [ATP] während der Versuche nicht wesentlich ändert. Die Abnahme der [ATP] erreicht noch nicht 1\%, wenn die [ATP] 9.10-3-m. ist, und wächst auf ungefähr $10 \%$ des Anfangswertes, wenn diese nur 1.10-3-m. beträgt.

Unter solchen Versuchsbedingungen zeigt sich, daß in Anwesenheit von ATP (Kurve 2, 3, 4, Abb. 3) die Aktomyosin-Löslichkeit gegenüber der Löslichkeit von ATP-freien Systemen beachtlich erhöht ist. Die Vergrößerung der Löslichkeit steigt mit der ATPKonzentration mindestens bis zu einer [ATP] $=9 \cdot 10^{-3}-m$. (Kurve 4). Dagegen verändert die An- 


\begin{tabular}{|c|c|c|c|c|}
\hline Nr. & $\begin{array}{c}\text { Ionen- } \\
\text { stärke } \\
\mu\end{array}$ & $\begin{array}{c}{[\mathrm{ATP}]} \\
10^{-3}-m .\end{array}$ & $\mathrm{Z}_{\eta}$ & $\frac{\Delta \mathrm{Z}_{\eta}}{\mathrm{Z}_{\eta}} \cdot 100$ \\
\hline 1 & 0,6 & - & 0,345 & 93 \\
2 & $\begin{array}{c}0,2 \\
0,2\end{array}$ & 8 & 0,174 & - \\
3 & $\begin{array}{c}\text { Extrakt 3 an- } \\
\text { schließend auf } \\
0,6 \mu \text { gebracht } \\
\text { derselbe nach } \\
\text { 24 Stdn. }\end{array}$ & $6-7$ & 0,2 & - \\
\hline
\end{tabular}

Tab. 1. Viskositätsvergleich einer Aktomyosin-Stammlösung $(0,6 \mu)$ mit dem Aktomyosin-Anteil, der bei $0,2 \mu$ in Anwesenheit von ATP in Lösung bleibt.

wesenheit von $0,003-0,03-m . \mathrm{Mg}^{++}$auch in ATPAnwesenheit die Löslichkeit (Kurve 3) ebensowenig wie in Abwesenheit von ATP (Abb. 1). Schließlich zeigt sich die Löslichkeit bei allen untersuchten ATPKonzentrationen von der Temperatur ebenso unabhängig (Kurve 2-4) wie ohne ATP. Die Gegenwart von PP in Konzentrationen von 1 bis $40^{\cdot} \cdot 10^{-3}-m$. ändert die normale Löslichkeit weder bei $0^{\circ}$ noch bei $20^{\circ} \mathrm{C}$ (vgl. Kurve 1, Abb. 3, mit den dazugehörigen Versuchspunkten).

Die Erhöhung der Aktomyosin-Löslichkeit durch ATP bei Ionenstärken von $0,1-0,2 \mu$ paßt nicht schlecht zu der Beobachtung, daß in dem gleichen Intervall der Ionenstärke sich die Aktomyosin-ATPase in die L-Myosin-ATPase verwandelt. Diese gilt umso mehr, als der größte Anteil des Aktomyosins jene niedrige Viskositätszahl zeigt $\left(Z_{\eta} \sim 0,2\right)$, die für das dissoziierte Aktomyosin oder L-Myosin charakteristisch ist (Tab. 1). Wenn das ATP gespalten ist, geliert der zunächst gelöste Anteil wieder. Wird diese Gelierung durch eine Ionenstärke von $0,6 \mu$ aufgehoben, so findet sich von neuem die hohe Viskositätszahl des undissoziierten Aktomyosins $(0,3-0,5)$ und eine entsprechende ATP-Empfindlichkeit (Tab. 1).

Doch beruht der Abfall der ATP-Spaltungsrate in höher konzentrierten ATP-Lösungen nicht ausschließlich darauf, daß sich ein Teil des Aktomyosins in gelöstes und dissoziiertes Aktomyosin verwandelt.

1. In den Spaltungsversuchen ${ }^{2}$ wird die Spaltungsrate des ATP bei einer Ionenstärke von $0,12 \mu$ bei $20^{\circ} \mathrm{C}$ sehr klein, obwohl unter diesen Bedingungen nur 60\% des Aktomyosins gelöst sind (Abb. 3).

2. Die Löslichkeit des Aktomyosin-Gels ist von der Temperatur und von der Gegenwart von $\mathrm{Mg}^{++}$ unabhängig. Die Umwandlung der Aktomyosin-
ATPase in die L-Myosin-ATPase wird dagegen durch fallende Temperatur und steigende $\mathrm{Mg}^{++}$begünstigt.

Es gibt also - besonders auffällig bei tiefer Temperatur und Anwesenheit von $\mathrm{Mg}^{++}$- einen $\mathrm{Zu}-$ stand des Aktomyosins, in welchem das Aktomyosin die fermentativen Eigenschaften des L-Myosins zeigt,

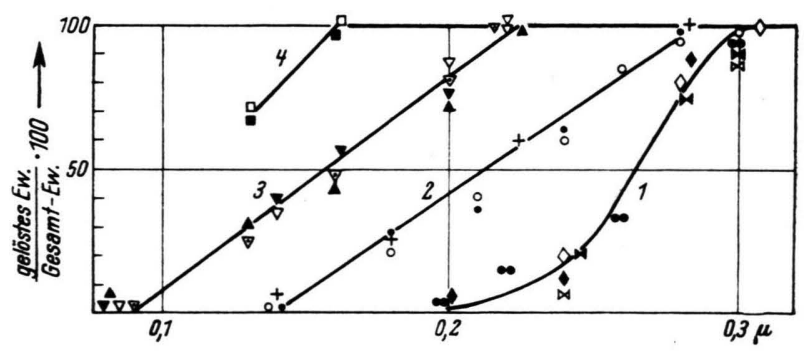

Abb. 3. Einfluß bei $20^{\circ}$ und $0^{\circ} \mathrm{C}$ von ATP und Pyrophosphat auf die Löslichkeit des Aktomyosins. Kurve 1: Löslichkeit in KCl-Lösungen mit und ohne Pyrophosphat, Kurve 2: in KCl-Lösung $+1 \cdot 10^{-3}-m$. ATP, Kurve 3: in $\mathrm{KCl}-L o ̈ s u n g ~+5 \cdot 10^{-3}-m$. ATP, Kurve 4: in KCl-Lösung $+9 \cdot 10^{-3}-m$. ATP. $\bullet 0,006 \mu$ Pyrophosphat, $\ \triangleright \triangleleft 0,06 \mu$ Pyrophosphat, $\bullet 0,06 \mu \mathrm{KCl}+$ Pyrophosphat. In allen Kurven gefüllte Symbole $20^{\circ} \mathrm{C}$, umrandete Symbole $0^{\circ} \mathrm{C}$. - 5.10-3-m. ATP $+3 \cdot 10^{-3}-m$. Mg, $\nabla 5 \cdot 10^{-3}-m$. АTP $+3 \cdot 10^{-3}-m$. Mg, + 1.10-3-m. ATP $+3 \cdot 10^{-2}-m$. Mg. Eiweißkonzentration: $0,1 \%, p_{\mathrm{H}}=7,0$.

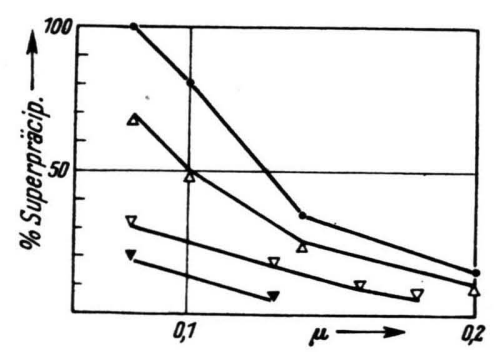

Abb. 4. Abhängigkeit der Superpräcipitation von der Ionenstärke, ATP- und Mg-Konzentration. \% Superpräcipitation $=$ Eiweißdichte in \% der maximalen Eiweißdichte, $\bullet$ in KCl-Lösung $+1 \cdot 10^{-3}-m$. ATP, $\Delta+3 \cdot 10^{-3}-m$. ATP, $\nabla 5 \cdot 10^{-3}-m$. ATP, $-+5 \cdot 10^{-3}-m$. ATP $+3 \cdot 10^{-3}-m$. $\mathrm{Mg}, \mathrm{T}=20^{\circ} \mathrm{C}, p_{\mathrm{H}}=7,0$.

obwohl sich das Aktomyosin im Gel und nicht im dissoziierten Solzustand befindet. Dies bedeutet, daß es 2 Zustände des Aktomyosin-Gels in Gegenwart von ATP gibt, die sich durch ihre fermentativen Eigenschaften unterscheiden.

Auf Grund früherer Beobachtungen ${ }^{2}$ liegt es nahe, den superpräcipitierten Zustand des Gels als den Zustand anzusehen, in dem das Gel die Eigenschaften einer Aktomyosin-ATPase hat. Es war bisher aber nicht bekannt, ob es bei Zimmertemperatur neben diesem Zustand - bei Gegenwart von ATP — über- 
haupt einen Zustand gibt, in dem das Gel nicht superpräcipitiert ist.

Abb. 4 zeigt, daß die Superpräcipitation des Gels mit zunehmender Ionenstärke sehr stark abnimmt,
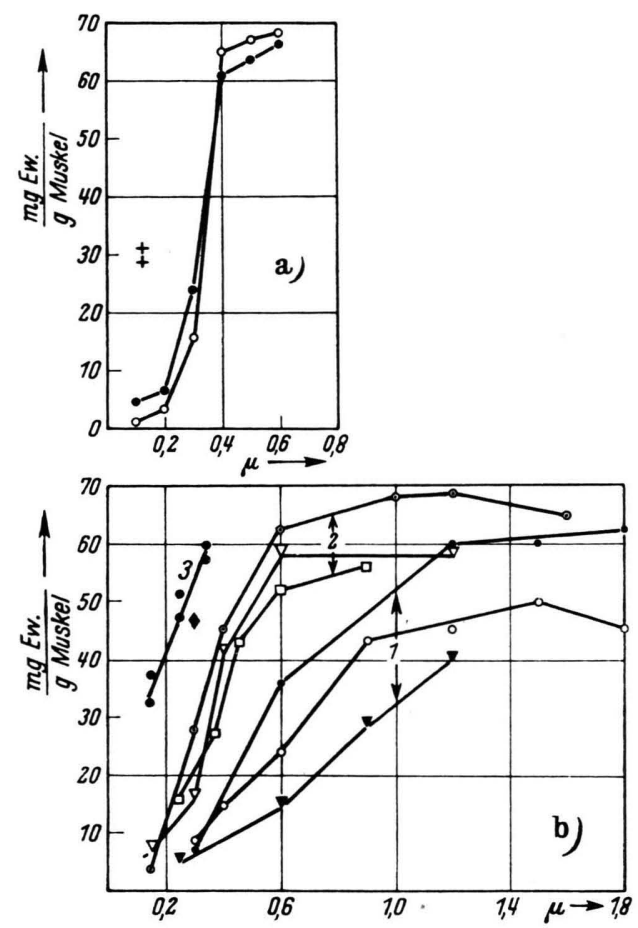

Abb. 5. Abhängigkeit der Myosinmenge, die aus Muskelfasern extrahiert werden kann, von der Ionenstärke und anderen Faktoren. a) Frischer Muskel: - Extraktion 15 Min. pm, 。 Extraktion 25 Min. pm, durch KCl-Lösung, $\ddagger$ durch KCl-Lösung $+6 \cdot 10^{-3}-m$. ATP, $0^{\circ} \mathrm{C}, p_{\mathrm{H}} \sim 6,5$, 2 Min. Blendorbehandlung, Extraktionsdauer 20 Min. b) Nach Vorextraktion mit 27-proz. Glycerin; Kurvengruppe 1: Extraktion durch KCl-Lösung, Kurvengruppe 2: durch KCl-Lösung $+1,6 \cdot 10-2-m$. Pyrophosphat, Kurve 3 durch KCl-Lösung $+6-8 \cdot 10-3-\mathrm{m}$. ATP. Überall: $\mathrm{T}=0^{\circ} \mathrm{C}$, $p_{\mathrm{H}} \sim 7,0 . \mathrm{Zu}$ Gruppe 1: Extraktionsdauer 1h. $\bullet$ Aufbewahrungszeit $0 \mathrm{~h}, 4$ Min. geblendort, $\circ$ Aufbewahrungszeit $126 \mathrm{~h}, 4 \mathrm{Min}$. geblendort, - Aufbewahrungszeit $102 \mathrm{~h}$, 2 Min. geblendort. Zu Gruppe 2: 4 Min. Blendorbehandlung, $\odot$ Aufbewahrungszeit $0 \mathrm{~h}, 1 \mathrm{~h}$ Extraktion, $\nabla$ Aufbewahrungszeit $120 \mathrm{~h}, 1 \mathrm{~h}$ Extraktion, o Aufbewahrungszeit 0 h, 20 Min. Extraktion. Zu Kurve 3: 4 Min. geblendort, Dauer der Extraktion 20 Min., - Aufbewahrungszeit $0 \mathrm{~h}$, Aufbewahrungszeit $120 \mathrm{~h}$. Die Dauer der Vorextraktion beträgt in allen Versuchen $5 \mathrm{~h}$ in 27 -proz. Glycerin.

in Anwesenheit von $5 \cdot 10^{-3}-m$. ATP und $\mathrm{Mg}^{++}$wird die Superpräcipitation Null (a), in Anwesenheit von $\mathrm{Mg}^{++}$bei einer Ionenstärke $\sim 0,14 \mu$ und in $\mathrm{Ab}$ wesenheit von $\mathrm{Mg}^{++}$bei einer Ionenstärke von $0,2 \mu$. Bei $0,14 \mu$ und einem ATP-Gehalt von $5 \cdot 10^{-3}-m$. ist das Aktomyosin nur zum kleineren Teil gelöst, aber überhaupt nicht mehr superpräcipitiert. Es gibt also in Gegenwart des ATP 3 Zustände des Aktomyosins: Superpräcipitiertes Gel, nicht-superpräcipitiertes Gel und schließlich dissoziiertes Sol. Die Ionenstärke, bei der der Übergang vom Gel zum Sol stattfindet, hängt allein von der ATP-Konzentration ab. Dagegen hängt die Ionenstärke, bei der der Übergang vom superpräcipitierten zum nicht-superpräcipitiertem Gel stattfindet, außerdem ab von der Konzentration der Erdalkalien und von der Temperatur. Das Intervall der Ionenstärke, in dem das Aktomyosin-Gel auch bei ATP-Gegenwart nicht superpräcipitiert ist, wächst mit abnehmender Temperatur und steigender ATP- und $\mathrm{Mg}^{++}$-Konzentration. Die Verwandlung der Aktomyosin-ATPase in die L-Myosin-ATPase ist dabei verbunden mit dem Verschwinden der Fähigkeit zur Superpräcipitation (vgl. Abb. 4 mit Abb. 5 von $\mathrm{H}$ as s e lb a c h${ }^{2}$ ).

$$
\text { V. }
$$

Die Löslichkeitsuntersuchungen an gereinigtem Aktomyosin sind aus technischen Gründen Löslichkeitsuntersuchungen an sehr wenig konzentrierten Eiweißsystemen. Es ist sicher, daß die ermittelten Daten für konzentrierte Aktomyosin-Systeme wie Muskelfasern oder Fibrillen nicht ohne weiteres gelten. Denn das muß keineswegs so sein: So findet die Umwandlung der Aktomyosin-ATPase in die LMyosin-ATPase in ATP-Gegenwart - ceteris paribus - erst bei wesentlich höherer Ionenstärke statt, wenn Muskelfibrillen mit einer Aktomyosin-Konzentration von $10-20 \%$ statt der gereinigten Aktomyosin-Systeme mit einer Konzentration von $1-2 \%$ verwendet werden ${ }^{3}$.

Ebenso ist es sicher, daß die Fibrillen einer lebenden Muskelfaser ungelöst und strukturiert sind, obwohl sie sich bei einer Ionenstärke von $\sim 0,2 \mu$ und einer ATP-Konzentration von $\sim 7 \cdot 10^{-3}-m$. unter Bedingungen befinden, unter denen die eiweißarmen gereinigten Aktomyosin-Systeme voll gelöst sind. Offen bleibt bei dieser Feststellung, ob in der biologischen konzentrierten Struktur wirklich die gegenseitige Affinität der Eiweißteilchen größer ist. Das könnte natürlich so sein. Es könnte aber auch so sein, daß nur die Dichte der Packung es dem einzelnen Eiweißteilchen unmöglich macht, so weit aus der Attraktionsphäre der anderen Teilchen herauszukommen, daß es beweglich wird. In letzterem Falle müßte sich die Fibrille von außen nach innen fortschreitend auflösen, wenn man sie in ein Bad gleicher Ionalität bringt. Wenn dagegen die gegenseitige 


\begin{tabular}{|l|c|c|c|c|}
\hline $\begin{array}{c}\text { Zer- } \\
\text { kleinerung }\end{array}$ & $\begin{array}{c}\text { Ionen- } \\
\text { stärke } \\
\text { !" }\end{array}$ & $\begin{array}{c}\text { [ATP] } \\
\cdot 10^{-3}-m .\end{array}$ & $\begin{array}{c}|c| \\
\text { Extraktionserfolg } \\
\text { Eyw. pro g } \\
\text { Muskel }\end{array}$ & $\begin{array}{c}\text { ATP-Emp- } \\
\text { findlichkeit } \\
\text { in \% }\end{array}$ \\
\hline 2' Blendor & 0,11 & - & 3,9 & 65 \\
$2^{\prime}$ Blendor & 0,11 & - & 6,8 & 61 \\
$2^{\prime}$ Blendor & 0,12 & 6 & 29,5 & 122 \\
$2^{\prime}$ Blendor & 0,12 & 6 & 31 & 102 \\
Latapie & 0,25 & - & 8 & 20 \\
Latapie & 0,25 & 6 & 12,5 & 22 \\
\hline
\end{tabular}

Tab. 2. Menge und ATP-Empfindlichkeit von Myosin, das bei niedrigen Ionenstärken mit und ohne ATP aus frischem Muskel extrahiert ist.

Affinität der Teilchen in der Fibrille größer wäre als in lockerem Gel aus gereinigtem Aktomyosin, müßte die Löslichkeit auch der Fibrille im Bade geringer sein als die des lockeren Gels.

Es lohnt also, die Löslichkeit der Myosin-Fraktion des Muskelbreis in Bädern abggestufter Ionenstärke und ATP-Konzentration zu messen und mit den entsprechenden Daten des gereinigten Aktomyosins zu vergleichen. Solche Messung ist aber nichts anderes als eine systematische Untersuchung der Extraktibilität des Myosins in Abhängigkeit von der Ionalität und der ATP-Konzentration. Sie hat deshalb auch praktische Bedeutung.

Für den frischen Muskel gilt: Ohne ATP-Zusatz zur Extraktionsflüssigkeit ist die Myosin-Extraktion praktisch vollständig bei Ionenstärke $>0,4 \mu$, falls der Brei in der Fleischmaschine und durch anschließende Blendor-Behandlung von 2 Min. zerkleinert ist. Bei einer Ionenstärke von $0,2 \mu$ ist - selbst wenn die Extraktion nur 7 Min. nach dem Beginn der Zerkleinerung anfängt - nie mehr als etwa $8 \%$ des Gesamtmyosins extrahiert (Abb. 5 a). Die Löslichkeit erscheint also etwas kleiner als die Löslichkeit gereinigten Aktomyosins unter vergleichbaren Bedingungen, d. h. bei Gegenwart von etwa $1 \cdot 10^{-3}-m$. ATP. Wird die ATP-Konzentration auf physiologischer Höhe gehalten, indem auch der Extraktionslösung $6 \cdot 10^{-3}-m$. ATP zugesetzt wird, so erhöht sich die Löslichkeit auf etwa den gleichen Wert wie beim gereinigten Aktomyosin-Gel; nämlich auf beinahe die Hälfte des gesamten Aktomyosins. Das gelöste Eiweiß erweist sich durch seine ATP-Empfindlichkeit und seine Viskosität in allen Fällen als Aktomyosin (Tab. 2). In Übereinstimmung mit früheren Erfahrungen ${ }^{6}$ gehen offenbar Aktin und L-Myosin bei

6 W. Hasselbach u. G. Schneider, Biochem. Z. 321, 462 [1951]. dem angewandten Zerkleinerungsverfahren in Lösung, ohne durch die Stroma-Struktur mechanisch wesentlich behindert zu werden. Wird der Muskelbrei nur mit dem Latapie hergestellt, so gehen in eine $7 \cdot 10^{-3}-m$. Extraktionslösung selbst bei einer Ionenstärke von $0,25 \mu$ nur $18 \%$ der Myosin-Fraktion über, und zwar als fast reines L-Myosin. Dies zeigt sich durch die geringe ATP-Empfindlichkeit im Original-Extrakt und Steigerung der der ATP-Empfindlichkeit durch die Aktin-Zusätze, die auf der Abszisse der Abb. 6 angegeben sind. Die geringe Dispersität

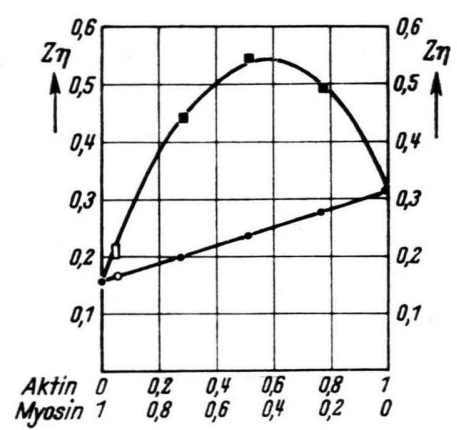

Abb. 6. Abhängigkeit der Viskositätszahl eines Myosinextraktes von der zugegebenen Aktinmenge. Extraktionsbedingungen: Frischer Muskelbrei, Latapie-zerkleinert, Ionenstärke $0,25 \mu, \mathrm{KCl}$-Lösung $+6 \cdot 10^{-3}-m$. ATP. $p_{\mathrm{H}} 6,5$. Versuchsbedingungen: Das umgefällte Myosin wird in $0,6 \mu \mathrm{KCl}$ gelöst, mit Aktin versetzt und auf seine Viskosität mit und ohne ATP untersucht. Eckige Symbole: ohne ATP. Runde Symbole: mit 3.10-4-m. ATP. Leere Symbole: extrahiertes Myosin. Gefüllte Symbole: extrahiertes Myosin + Aktin. Durch Extrapolation der Kurve bis zu dem Schnittpunkt ergibt sich für den Extrakt ein Verhältnis Myosin zu Aktin $~ 95: 5$.

des Muskelbreis hindert den Austritt des Aktins offenbar fast vollständig und den des L-Myosins weitgehend. Bei hoher Ionenstärke $(0,6 \mu)$ ist bekanntlich das L-Myosin auch bei Latapie-Zerkleinerung im Gegensatz zu Aktin vollständig extrahierbar. Worauf dieser Unterschied beruht, wurde bisher noch nicht untersucht.

Es sieht also so aus, als sei die gegenseitige Affinität der Aktomyosin-Teilchen im frischen Muskel und im Gel aus gereinigtem Aktomyosin nicht allzu verschieden. Daß trotzdem unter physiologischen Normalbedingungen die Fibrille ein Gel und das gereinigte Aktomyosin ein Sol ist, dürfte vorwiegend auf der verschiedenen Konzentration des Aktomyosins beruhen.

Dagegen nimmt die gegenseitige Affinität der Aktomyosin-Teilchen deutlich zu, wenn das mit Blendor behandelte Material mit 27-proz. Glycerin 
vorextrahiert wird: Bei einer nachfolgenden Extraktion mit KCl-Lösung allein $p_{\mathrm{H}} \sim 7$ ) wird das Aktomyosin erst bei einer Ionenstärke $\geq 1,2 \mu$ annähernd vollständig extrahiert. Wenn das Präparat hinterher noch 5 Tage in $\sim 80$-proz. Glycerin aufgehoben wird, ist die Extraktion selbst bei diesen hohen Ionenstärken nicht ganz vollständig. Dies gilt auch dann, wenn die Extraktionszeit auf 1 Stde. ausgedehnt wird. Außerdem ist die Ausbeute vergrößert, wenn die Blendor-Behandlung auf 4 Min. verlängert wird. Bei einer Ionenstärke von $0,4 \mu$ tritt auch dann nur etwa ein Viertel des vorhandenen Aktomyosins in die Lösung über (Abb. 5 b, Kurvengruppe 1).

Dieses Anwachsen der Kohäsionskräfte zwischen den Eiweißteilchen beruht sicher zu einem sehr erheblichen Teil darauf, daß die Präparationen weder ATP noch anderes Polyorthophosphat enthalten, das eine Dissoziation des Aktomyosins bei höheren Ionenstärken zu bewirken vermag. Denn auch frischer Muskelbrei aus totenstarren Muskeln oder Muskelbrei, aus dem das ATP ausgewaschen wurde, geben bis zu beträchtlichen Ionenstärken $(0,5 \mu)$ kein Aktomyosin mehr $a b^{\overline{7}, 8}$.

Immerhin spricht die Tatsache, daß die Präparate, die nach 5-stdg. Extraktion in 27-proz. Glycerin sofort extrahiert werden - wenigstens bei höheren Ionenstärken - etwas mehr Aktomyosin abgeben als nach 5-tägig. Aufbewahrung in 80-proz. Glycerin dafür, daß zusätzlich auch noch ein Alterungsfaktor wirksam wird (Abb. 5 b, Kurvengruppe 1).

Werden solche mit Glycerin-Wasser extrahierten Präparate in Anwesenheit von 0,016-m. PP extrahiert, so wächst die extrahierte Myosinmenge beträchtlich. Der Unterschied zwischen 5 Tage alten und sofort extrahierten Präparaten wird so klein, daß er nicht mehr gesichert erscheint, und auch Verminderung der Extraktionsdauer von 1 Stde. auf 20 Min. ändert am Ergebnis nicht viel. Die Extraktionsausbeute ist fast dieselbe wie bei der Extraktion frischen Muskels (Abb. 5 b, Kurvengruppe 2).

Wird soviel ATP zugegeben, daß die ATP-Konzentration am Schluß noch immer 6-7 $10^{-3}-m$. ist, so steigt die Ausbeute an Myosin ungefähr auf denselben Betrag wie beim frischen Muskel unter dem gleichen ATP-Zusatz (Abb. 5b, Kurve 3).

Es genügt also eine Durchtränkung Wasser-Glycerin extrahierter Fasern mit ATP von physiologischer Konzentration, um in 20 Min. die Kohäsionskräfte

7 M. Dubuis son u. L. Mathieu, Experientia [Basel] 4, 152 [1948].

C P. Crepax, Biochim. biophysica Acta 7, 87 [19.51]. und die Löslichkeit des Aktomyosins auf den Normalwert zurückzuführen. Die große Abnahme der Löslichkeit durch die Wasser-Glycerin-Extraktion ist also in den Fehlergrenzen der Löslichkeitsbestimmung voll reversibel. Das gleiche gilt für die geringe zusätzliche Löslichkeitsabnahme, die der Extraktion in den nächsten $100 \mathrm{Stdn}$. folgt.

Mit ATP hängt die Löslichkeit der Wasser-Glycerin extrahierten Fasern ungefähr ebenso von der Ionenstärke $a b$, wie die Löslichkeit des eiweißarmen Gels aus gereinigtem Aktomyosin (vgl. Abb. 5b mit Abb. 3). Ohne ATP oder andere Polyorthophosphate ist die Löslichkeit der extrahierten Fasern bis zu einer Ionenstärke von $1,2 \mu \mathrm{sehr}$ viel schlechter. Es ist möglich, daß dies nur auf der verschiedenen Methodik beruht: An den Fasern wird geprüft, wieviel Myosin noch in Lösung geht, wenn das Myosingel der Faser lange Zeit in ATP-freien Lösungen niedriger Ionenstärke aufbewahrt war; am gereinigten Aktomyosin wird dagegen geprüft, wieviel Aktomyosin ausfällt, wenn eine Aktomyosin-Lösung in eine ATP-freie Lösung gleicher niedriger Ionenstärke hineingegeben wird. Im zweiten Fall bleibt also für eine nachträgliche Verfestigung des Niederschlages keine Zeit.

Der Einfluß des PP auf die Löslichkeit des FaserAktomyosins ist qualitativ schon länger bekannt und wichtig als Hilfsmittel bei der Extraktion ${ }^{6}$. Dieser Einfluß des PP auf das Faseraktomyosin ist geringer als der Einfluß des ATP. Auf der anderen Seite wird die Abhängigkeit der Fällung gereinigten Aktomyosins von der Ionenstärke durch PP nicht beeinflußt. Beide - scheinbar sich widersprechenden Beobachtungen lassen sich zusammenfassen in der Vorstellung: Das PP verhindert nicht, daß das Aktomyosin bei niedrigen Ionenstärken unlöslich wird, aber es vermag die anschließend im Gel auftretenden Alterungs- und Verfestigungserscheinungen wieder aufzuheben.

\section{Beschreibung der Versuche}

Eisgekühlte Kaninchenmuskulatur wurde nach Zerkleinerung mit der gekühlten Fleischmaschine 4 Min. im Blendor in der dreifachen Menge kalter $0,6-m . \mathrm{KCl}$ zerkleinert und 24 Stdn. im Eisschrank extrahiert. Nach der ersten Umfällung des Extraktes bei 0,05 $\mu$ wurde zur Entfernung von überschüssigem L-Myosin 2-mal bei $0,25 \mu$ fraktioniert. Im Anschluß an die Fraktionierung wurde noch 2 -mal bei $0,05 \mu$ umgefällt. Die etwa 1-proz. Aktomyosin-Stammlösung wurde auf $0,4-m . \mathrm{KCl}$ und $p_{\mathrm{H}} \sim 7$ gebracht.

Die Bestimmung der Löslichkeit des Aktomyosins in Abhängigkeit von der Ionalität des Lösungsmittels wurde in $10 \mathrm{ccm}$ fassenden Zentrifugengläsern ausgeführt. 
Mẹnge und Ionalität des Lösungsmittels wurden so gewählt, daß sich nach Zugabe von 0,5-1 ccm der Aktomyosin-Stammlösung in der Lösung die gewünschte Ionalität und Eiweißkonzentration einstellte. Das ungelöste Eiweiß wurde durch 3 Min. dauerndes Zentrifugieren von dem gelösten Eiweiß abgetrennt. Der Eiweißgehalt einer aliquoten Menge der überstehenden Lösung wurde nach Trichloressigsäurefällung und Auswaschen des ATP als Mikrokjeldahl bestimmt und hieraus die Menge des im Gesamtvolumen gelösten Eiweißes berechnet.

Bei der Untersuchung des Einflusses des ATP auf die Löslichkeit des Aktomyosins waren eine Reihe besonderer Vorkehrungen notwendig, da das Aktomyosin besonders bei niedrigen Ionenstärken und niedrigen ATP-Konzen-

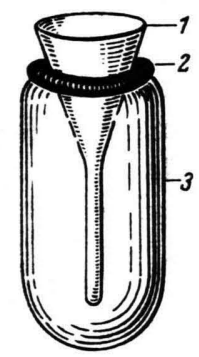

Abb. 7. Zentrifugenglas zur Messung der Superpräcipitation. 1. Reaktionsgefäß mit Meßkapillare, 2. Gummiring, 3. Trägerglas.

trationen den ATP-Gehalt des Ansatzes durch Spaltung sehr schnell verringert.

1. Die ATP-haltigen Salzlösungen wurden erst unmittelbar vor dem Beginn des Zentrifugierens mit Aktomyosin beschickt und vermischt, so daß bis zur Beendigung des Zentrifugierens und Abtrennens des gelösten vom ungelösten Eiweiß nie mehr als 4 Min. verstrichen.

2. In den Ansätzen mit besonders hoher ATPase-Aktivität des Aktomyosins wurde die Eiweiß-Konzentration herabgesetzt und die Konzentration der ATP-Lösung so gewählt, daß in den 4 Min. bis zur Abtrennung des in Lösung gebliebenen Eiweißes die gewünschte ATP-Konzentration um nie mehr als $10 \%$ unterschritten wurde.

Die quantitative Messung der Superpräcipitation erfolgte in den abgebildeten Zentrifugenröhrchen (Abb. 7). In dem kegelförmigen Oberteil, der $5 \mathrm{ccm}$ faßt, wird das Aktomyosin mit der ATP-haltigen Salzlösung vermischt. Die Eiweißmenge wurde so gewählt, daß der Verlust an ATP durch die ATP-Spaltung während des ebenfalls 4 Min. dauernden Versuches zu vernachlässigen war und das Volumen des nicht superpräcipitierten Gels das Volumen der Kapillare nicht überschritt. Aus der Höhe der Eiweißsäule kann nach Korrektur für den gelösten Anteil des Aktomyosins auf den Grad der Superpräcipitation geschlossen werden. Als Maß für die Superpräcipitation wird die relative Eiweißdichte, bezogen auf die maximale Eiweißdichte, bei vollständiger Superpräcipitation angegeben.

\section{Die Bestimmung der Extrahierbarkeit des frischen Muskels}

Zur Bestimmung der Extrahierbarkeit des frischen Muskels bei verschiedenen Ionenstärken wurde $1 \mathrm{Tl}$. des gekühlten Muskels mit $3 \mathrm{Tln}$. von $0,15-m$. $\mathrm{KCl}$ von etwa $0^{\circ} 2$ Min. im Blendor zerkleinert. In $1 \mathrm{Tl}$. Extraktionsflüssigkeit $\left(0^{\circ}\right), p_{\mathrm{H}}=6,5$, wurden 4 Tle. der Fasersuspension einpipettiert. Die Angaben über die Ionalität beziehen sich immer auf die Mischung. Die Ansätze ohne ATPZusatz zur Extraktionsflüssigkeit wurden 20 Min., die mit ATP-Zusatz (6-7.10-3-m.), um den Verlust der Spaltung zu verringern, nur $10 \mathrm{Min}$. extrahiert. Bestimmung und Berechnung des extrahierten Myosins erfolgte wie bei $\mathrm{H}$ a s s elb a ch und $\mathrm{S} c \mathrm{hneider}$.

$$
\begin{gathered}
\text { Die Bestimmung der Extrahierbarkeit } \\
\text { des mit Glycerin-Wasser } \\
\text { extrahierten Muskels }
\end{gathered}
$$

Frischer gekühlter Muskel wurde in kalter 27-proz. Wasser-Glycerin-Lösung 2-mal 2 Min. geblendort. Nach 5 Stdn. wurde der vorextrahierte Muskelbrei abzentrifugiert, dann wurden entweder die gelösten wasserlöslichen Eiweißkörper sofort ausgewaschen und anschließend aus abgewogenen Mengen das Myosin bei $p_{\mathrm{H}} 6,7-7,0$ extrahiert, oder der Brei wurde in $\sim 80$-proz. Glycerin im Eisschrank aufgehoben. Nach 4-5 Tagen wurde nach Verdünnen des Glycerins mit Wasser der Muskelbrei abzentrifugiert, wiederholt mit Wasser gewaschen und dann ebenfalls abgewogene Breimengen bei verschiedenen Ionenstärken extrahiert. Das Verhältnis Faserbreimenge zu Extraktionsflüssigkeit betrug etwa 1:10. Die Extraktion erfolgte bei $0^{\circ} \mathrm{C}$ und dauerte bei der KCl-Extraktion 1 Stunde. Die Ansätze mit ATP wurden immer, die Ansätze mit PP-Zusatz nur in einer Versuchsreihe $20 \mathrm{Min}$. extrahiert. In den ATP-haltigen Ansätzen wurde zurVermeidung zu hoher Spaltungsverluste das Verhältnis Faserbreimenge zu Extraktionsflüssigkeit auf 1:20 herabgesetzt.

Das Myosin wurde aus aliquoten Teilen des Extraktes bei $0,03 \mu$ ausgefällt und kjeldahlometrisch bestimmt.

Die viskometrische Charakterisierung der Eiweißkörper erfolgte im Ubbelohde-Viskosimeter. Berechnung der spezifischen Viskosität und der ATP-Empfindlichkeit nach Portzehl, S chramm u. Weber 1 .

Das in den Versuchen verwandte Na-ATP wurde aus Ba-ATP hergestellt, das uns die Firma $\mathrm{H}$ e $\mathrm{n} n$ in $\mathrm{g}$ dankenswerterweise zur Verfügung gestellt hat. 